\title{
From Humans to Robots: the Role of Cutaneous Impairment in Human Environmental Constraint Exploitation to Inform the Design of Robotic Hands
}

\author{
Giuseppe Averta ${ }^{1,2}$, Cosimo Della Santina $^{1}$, Edoardo Battaglia ${ }^{1}$, \\ Simone Ciotti ${ }^{1,2}$, Visar Arapi ${ }^{1}$, Simone Fani ${ }^{1,2}$, and Matteo Bianchi ${ }^{1,3}$ \\ ${ }^{1}$ Centro di Ricerca "E.Piaggio" - Università di Pisa, Pisa, Italy \\ ${ }^{2}$ Fondazione Istituto Italiano di Tecnologia, Genova, Italy \\ ${ }^{3}$ Department of Information Engineering, University of Pisa, Pisa, Italy \\ Corresponding Author: g.averta3@gmail.com
}

\begin{abstract}
Human hands are capable of a variety of movements, thanks to their extraordinary biomechanical structure and relying on the richness of human tactile information. Recently, soft robotic hands have opened exciting possibilities and, at the same time, new issues related to planning and control. In this work, we propose to study human strategies in environmental constraint exploitation to grasp objects from a table. We have considered both the case where participants' fingertips were free and with a rigid shell worn on them to understand the role of cutaneous touch. Main kinematic strategies were quantified and classified in an unsupervised manner. The principal strategies appear to be consistent in both experimental conditions, although cluster cardinality differs. Furthermore, as expected, tactile feedback improves both grasp precision and quality performance. Results opens interesting perspective for sensing and control of soft manipulators.
\end{abstract}

Index Terms-Grasp; Haptic Impairment; Human and robot hands; Manipulation.

\section{INTRODUCTION}

Hands are our preeminent tool to interact with, manipulate and explore the environment. A lot of effort has been hence devoted to study human hands and try to model and replicate their behavior for the design and control of robotic systems [1], [2].

Not surprisingly, grasping and manipulation represent two key topics in robotics [3]. Recently, we have observed a change of perspective in robotic hands: from rigid devices, to systems that can deform and adapt to the external environment in a new framework called soft manipulation. This can be achieved through a continuous deformable mechanical structure, as for RBO hand [4] in which fingers are made by a deformable material, or by introducing passive elastic elements in the design, as done for the Pisa/IIT SoftHand [5]. In the latter approach, softness is guaranteed by elastic elements in joints while maintaining rigidity at fingertips level.

Soft manipulation paradigm has opened new opportunities for grasping. Indeed, environmental constraints are no more

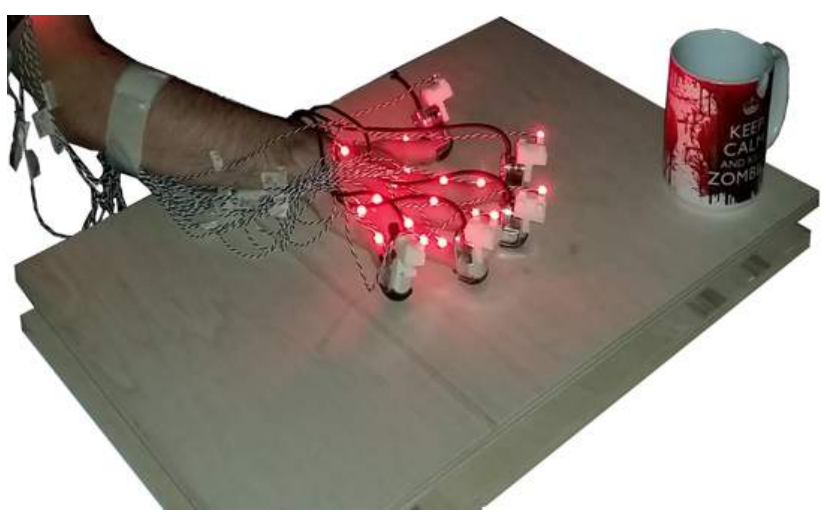

Fig. 1. Hand wearing ThimbleSense and optical markers supported by the sensorized table.

regarded as constraints per se but as enabling factors to multiply grasping capabilities as humans actually do [6]. Under a control point of view, the planning phase is completely changed. Rigid hands require finger trajectories that can bring the fingertips in specific positions while avoiding contacts of the hand with the environment. Soft manipulation, instead, subverts this scheme. Hand-object, object-environment and hand-environment contacts are not avoided but rather sought after and exploited to shape the hand itself around the object [3], [7].

Since this is similar to what humans do, a successful strategy to advance soft manipulation planning could be the observation of humans to devise planning strategies. Previous studies of our group focused on upper limb main movements during daily living activities [8], in this work we inspect the principal human grasping approaches for impaired and unimpaired tactile conditions. For the former case, tactile feedback is impaired requiring subjects to wear rigid shells on the fingertips (see Fig. 1), which creates a situation that is similar to the case of 
robotic hands with rigid pads but compliant structures. The shells that we used are a sensorized system called ThimbleSense [9], which provides 6-axis force and torque measurements for each fingertip, as well as the location of the contact centroid generated by tactile interaction. We performed experiments with human participants grasping a set of common objects from a table: hand movements were recorded through a motion capture system, and the contact forces exerted on the environment were recorded through a sensorized table. Then we identified few principal clusters of hand configurations adopted by humans during environmental constraint exploitation (ECE), using unsupervised clustering techniques.

Outputs of our analysis reveal a good consistency of hand configurations between the impaired and unimpaired case but changes in cluster cardinality. What is noticeable is that, for the impaired case, the grasp is generally less precise. Moreover, looking at contact forces and contact time values, we found that the impaired case present higher contact forces with the environment and requires an higher amount of time to complete the task.

We believe that our findings suggest the importance of a sensorization for robotic soft hands, e.g. through IMU for contact detection, and could inspire planning strategies for grasping, manipulation and ECE. Future works, discussed in the conclusions of this paper, will further inspect the applications of these results for sensing and planning of soft robotic hands. This paper is organized as follow: first, a section Materials and Methods shows the experimental setup, the experiments performed for this work and the software tool for data extraction; second, the section Data Analysis and Results contains the analysis post-experiments performed on the data and draw some conclusions; finally, the section Conclusions and Future Works capitalizes on the main findings of this works opening fascinating perspective for future works.

\section{Materials And Methods}

\section{A. Participants}

We asked six right-handed able-bodied volunteers (three females, three males; age range: 23-27 years, mean 25.17 years) to reach and grasp an object from a table. All subjects were absolutely unaware regarding of the experimental purpose of the study and had no history of neuromuscular disorders. Each participant signed an informed consent to participate in the experiment and the experimental protocol were approved by the Institutional Review Board of University of Pisa, in accordance to the declaration of Helsinki.

\section{B. Experimental Setup}

1) Hand sensorization: For the experiments of this work we used a multi-modal acquisition setup based on a optical Motion Capture system with active markers (Phase Space).

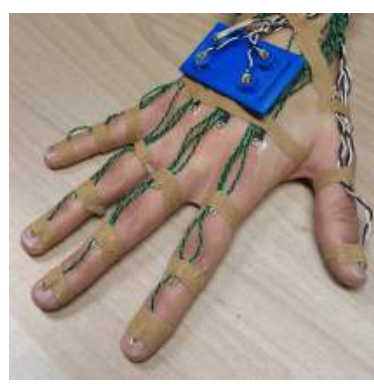

(a) Hand markerization.

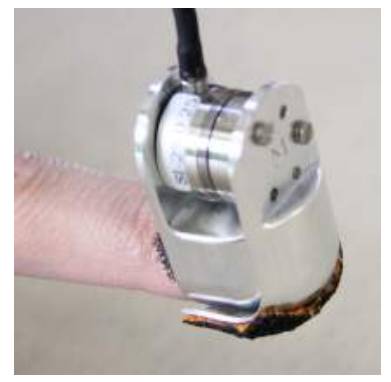

(b) ThimbleSense shell.

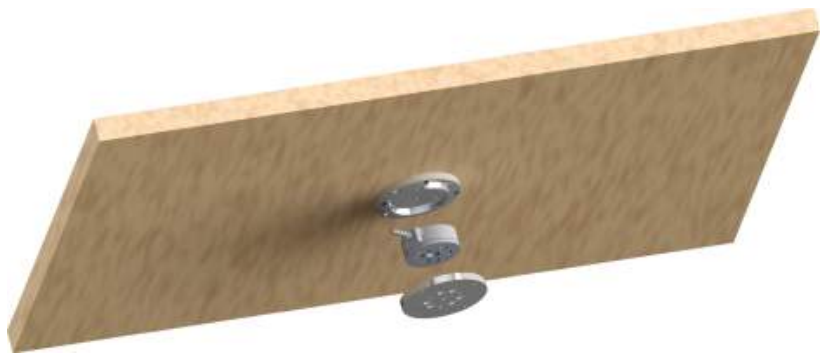

(c) Sensorized table.

Fig. 2. a) 24 markers are fastened to hand and phalanges for hand kinematic tracking. b) The tactile impairment is obtained by wearing ThimbleSense shells on fingertips. $c$ ) Exploded view of the sensorized platform used in the experiments.

Ten stereo-cameras working at $480 \mathrm{~Hz}$ tracked 3D positions of 24 markers fastened to hand and phalanges as shown in Figure 2a. In the impaired case, the tactile impairment was guaranteed by asking subjects to wear shells of ThimbleSense on fingertips (see Figure 2b). ThimbleSense is a wearable sensing system that provides force/torque data and position of the contact centroid on each fingertip [9]. Force and torque measurements from each fingertip were recorded and will be used for future analysis. A preliminary investigation of the effect of ThimbleSense on tactile feedback was carried in [9].

2) Sensorized Table: For the analysis of exchanged forces between the subjects hand and the environment, we used a sensorized platform, which includes a force-torque sensor ATI mini45 (see http://www.ati-ia.com/products/ft/ft_models.aspx? $\mathrm{id}=$ Mini45 for specifications) mounted as in Figure 2c. The sensor is used to measure interaction forces/torques with the table after a contact.

3) Scene Recording: Two cameras (Logitech hd 1080p) were also used to record the experiments from two viewpoints to keep track of the whole execution. We used blue filters to improve the recordings quality, which would otherwise have been reduced by the poor light conditions and the interference of red light emittance from the active markers of the motion capture system. 


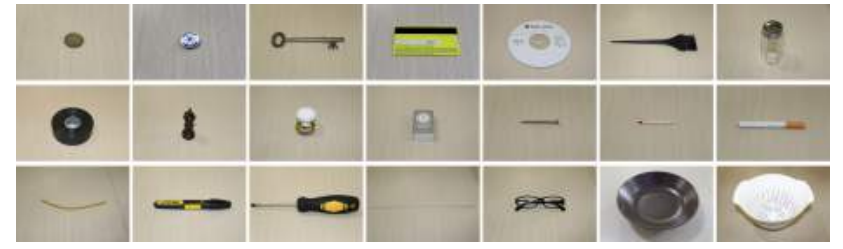

Fig. 3. The set of 21 common use object used for the experiments.

4) Objects Set: We asked to grasp and interact with a set of 21 common objects. The set was composed by the following items: 2 euro coin, 1 button badge, key, credit card, CD, comb hair color, salt shaker, tape, chessman (queen), knob, matchbox, screw, match, cigarette, rubber band, marker, screw driver, shashlink, glasses, coffee mug, plat (see Fig. 3). In contrast with previous work on grasping real objects (e.g. [10]), we chose objects that are difficult to grasp and therefore necessitate exploitation of the flat surface constraint. A similar set of objects was used in [11] for an analogous table-top scenario, to elicit a rich set of ECE strategies.

\section{Setup Harmonization}

The acquisition was implemented through a customized application developed in C++ employing i) Boost libraries [12] to perform the synchronization between Phase Space data and force/torque sensors, ii) Phase Space OWL library to get the optical tracking system data iii) a custom library providing an interface to acquire the force/torque sensor data from the table [13]. The acquisition system allows to organize and synchronize data with respect to an absolute clock with period $0.025 \mathrm{~s}$.

\section{Experiment}

The experiments were designed with the aim to analyze human behavior in grasping and manipulation tasks involving ECE.

Participants were asked to comfortably sit in front of the table, as depicted in Figure 4 and was instructed to pose their hand in a specific initial position, drawn at the right side of the sensorized surface as in Figure 4b. The distance between the hand starting position and the site where the object was placed was $60 \mathrm{~cm}$. For each trial, participants were asked to reach the object posed in the center of the sensorized surface. Once the hand reached the object, subjects were asked to grasp the object, lift (20 cm height), hold (1 sec), put it back on the table and place the hand back in its starting position. The experimenter gave the start signals to subjects. While imparting instructions, the experimenter emphasized that the whole movement should have been performed in a natural fashion, i.e. as if the subject was about to use it, in accordance with [14]. Two trials were performed for each of the 21 objects. Object order was randomized for each subject. The sequence

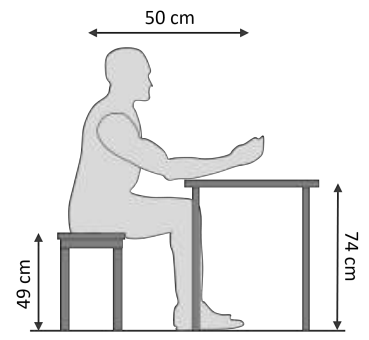

(a) Setup side view.

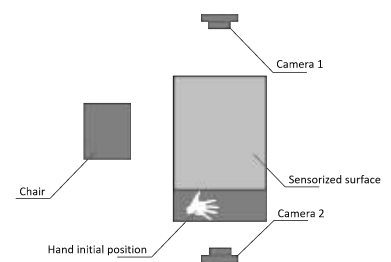

(b) Setup top viewl.
Fig. 4. Table-Subject disposition.

of experiments was repeated two times, with and without tactile impairment, for a total of 84 trials per subject. Each subject performed the whole experiment in a single day. The experiments with tactile impairment were performed in the morning, while the experiments without tactile impairment in the afternoon, as in [9].

\section{E. Kinematic Model and Angles Estimation}

An accurate description of the human hand is challenging due to the high number of bones and joints composing the human hand. As a trade-off between accuracy and complexity, in this work we considered a 20 DoF kinematic model (see Fig. 5).

Each long finger is described by a set of four angles: two DoFs for flexion-extension and abduction-adduction in metacarpophalangeal joints, one DoF for flexion-extension in proximal and distal intra-phalangeal joints. The thumb is described with four angles: two DoFs for the trapeziometacarpal, one DoF for the metacarpo-phalangeal, and one DoF for the interphalangeal. To reconstruct realistic values of the joint angles from marker data, we implemented an identification procedure of the model parameters, as in [15]. Data for the calibration procedure were acquired by asking subjects to perform the Kapandji test [16],
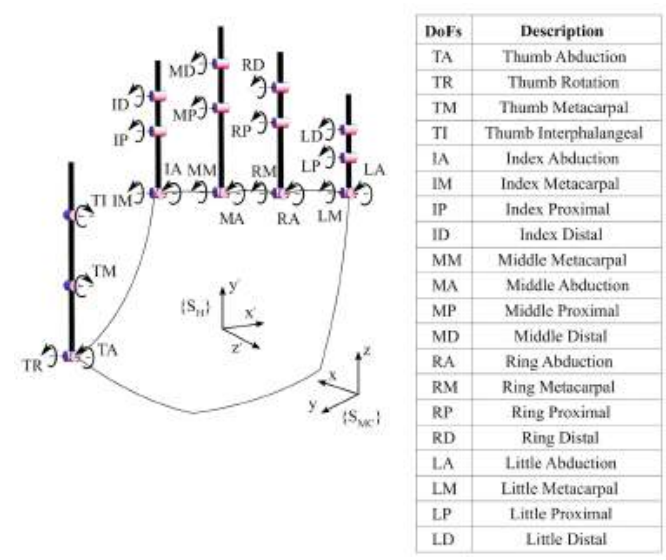

Fig. 5. Kinematic model of the hand. 
i.e. touching the four long fingers with the tip of the thumb. The test was repeated two times. The parameters to be identified were: $a_{B} \in R^{30}$ collecting the length of each phalanx and the three space positions of the abduction joints (TR/TA, IM/IA, MM/MA, RM/RA e LM/LA in Fig. 5) w.r.t. the local frame, and $a_{G} \in R^{45}$ collecting the location of each marker placed on the phalanxes w.r.t. the joint to which the LED is connected. Their values are calculated minimizing the Root Mean Square Error (RMSE) between measured marked positions $y_{k}$ and the estimated ones from the hand kinematics $f\left(x_{k} ; a_{G}, a_{B}\right)$, in a set of reference time stamps $K_{i d}$, i.e. by solving the following problem

$$
\begin{aligned}
\arg \min _{x, a_{G}, a_{B}} \sqrt{\sum_{k \in K_{i d}}\left(y_{k}-f\left(x_{k} ; a_{\mathrm{G}}, a_{\mathrm{B}}\right)\right)^{T}\left(y_{k}-f\left(x_{k} ; a_{G}, a_{B}\right)\right)} \\
\text { s.t. } x_{k}>0 \forall k
\end{aligned}
$$

where $x_{k} \in R^{20}$ is the vector collecting the estimation of joint angles at the $k$-th time step, $x_{k}>0$ is to be considered element-wise, $x$ is the vector collecting all the joint estimations in the considered time steps. We selected $K_{i d}$ as 60 temporally equidistant frames. The constraint $x_{k}>0$ accounts for biomechanical joint limits, to achieve a more robust estimation and to reduce local minima [15].

The optimization problem is solved through the MatLab function fmincon. The initial guess for the parameters $a_{G}$ and $a_{B}$ are evaluated through direct measurements, carried out with a caliper on each subject's hand before the experiment (the initial value of $x$ is the null vector). Articulated hand postures typically produce marker occlusion to cameras. We used here Piecewise Cubic Hermite Interpolating Polynomials [17] to interpolate missing values. Once estimated model parameters, to obtain the hand postures from marker position we used a Constrained Extendend Kalman Filter [18]. Kalman filtering was already employed for a similar scope in [19]. We rely on the identified hand model, representing joint evolution as a random walk. In each time step the filter estimates hand posture from the measure of the marker position in space and the previous state estimation. Joint limits are considered as constraints.

To increase the robustness against marker losses (we had an average of 2.47 marker loss per frame, and a maximum number of consecutive marker loss of $\sim 15$ ), we multiply at each step the Kalmann Filter observation noise covariance matrix by the number of consecutive missing measures. The filter was initialized with the open hand posture, and a null state covariance matrix. The observation noise covariance matrix is $0.001 I_{20 \times 20}$ and the state noise covariance matrix is $0.0005 I_{24 \times 24}$. Both the matrices were heuristically tuned. The reconstruction quality was evaluated as the RMSE between measured and estimated LED positions. The mean values is
$2.9 \mathrm{~mm}$ for the unimpaired case and $3.2 \mathrm{~mm}$ for the impaired case.

\section{F. Pre-processing of Force Data}

Force/torque data from ATI Mini45 (i.e. sensorised surface) are filtered through a moving average filter based on SavitzkyGolay method [20]. The window width was the $1.5 \%$ of the total data length. We then used the known dimensions of the surface to evaluate the centroid of contact with the table through the tactile toolbox, as in [21]. A portable version of this tool, as raw data of our experiments, will be made freely available through handcorpus ${ }^{1}$, an open-access on-line repository for grasping experimental data on human and robot hands.

\section{Data Analisis And Results}

\section{A. Unsupervised clustering of hand configuration}

To inspect human grasping strategies we selected, for each subject and for each task, the first frame of contact. This frame was obtained looking at force data from the sensorized table, as the one for which the norm of force exceeded a threshold $(0.1 \mathrm{~N})$. To increase clustering robustness and include also the configuration after contact, we selected also a second frame, one second after the first one. After this selection we used k-means algorithm as unsupervised clustering with the goal to find principal hand configurations for the contact frame and the delayed frame. We found that for both the impaired and unimpaired case three clusters allow to increase the compactness of clusters by more than $80 \%$ (see Fig. 6).

${ }^{1}$ www.handcorpus.org

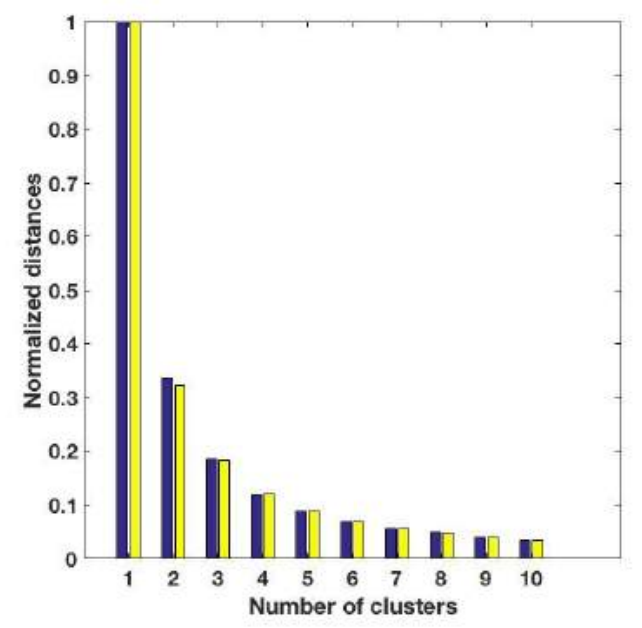

Fig. 6. Mean values of the within-cluster sums of point-to-centroid distances w.r.t. the number of clusters used. On blue the unimpaired case, on yellow the impaired case. 

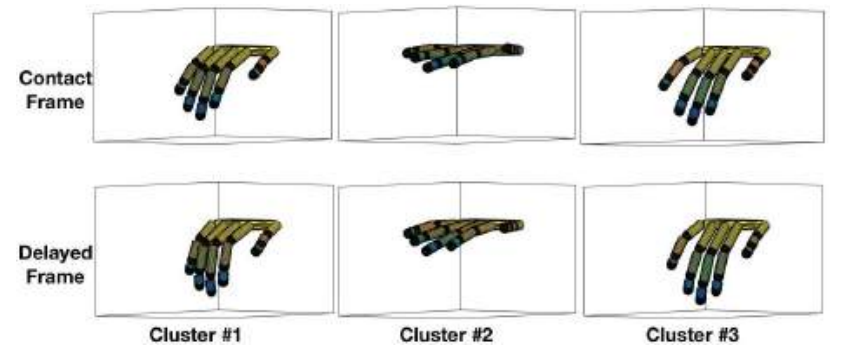

(a) Clusters unimpaired case.
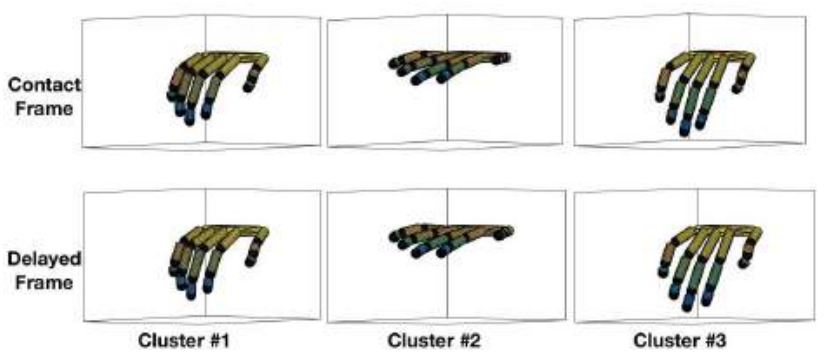

(b) Clusters impaired case.

Fig. 7. Centroids of the three principal clusters of hand configurations in impaired and unimpaired cases. For each one we report the configuration in the contact time frame and the configuration in a time frame delayed by $1 \mathrm{sec}$. In both the conditions we can identify one cluster for power grasp (Cluster \#1), one cluster for sliding configuration (Cluster \#2) and one cluster for precision grasp (Cluster \#3).

Both for the unimpaired and impaired case, we identified three main hand configurations:

- Power grasp (Cluster \#1), characterized by higher closure of the long fingers;

- Sliding configuration (Cluster \#2), in which fingers are spread out along the table;

- Precision grasp (Cluster \#3), with long fingers slightly extended.

The centroids of the three clusters are plotted in Figure 7. We found that for the unimpaired case the cardinality of the first cluster is 68 , the cardinality of the second cluster is 92 and the cardinality of the third cluster is 82 . For the impaired case, instead, the cardinality of the first cluster is 80 , the cardinality of the second cluster is 117 and the cardinality of the third cluster is 43 . What is noticeable is that for the latter case we found an increase of sliding and power grasp approaches. This suggests that precision grasp is penalized by the absence of tactile feedback. Under a robotic point of view, this result can suggest main planning strategies for ECE with soft robotic hands but also can inform the design of robotic hands sensorization. For example, IMUs could be used, as a first approximation of haptic information, to detect contacts with the environment and to be employed in refining and completing the ECE strategies reported in the previous section, e.g. as a combination of feedback and feedforward actions.

\section{B. Analysis of contact forces and times of task execution}

The ATI sensor placed under the table allowed to measure the force/torque applied by the subject while interacting with the environment. We found that subjects with tactile impairment required more time to complete the task w.r.t. the unimpaired case. In particular, the complete task is performed in $13.9 \pm$ $2.7 \mathrm{~s}$ for the tactile impairment case, while it is performed in $11.5 \pm 2.0 \mathrm{~s}$ in the other case. Also the amount of time required for the interaction with the table increased for the tactile impairment case. In fact, subjects with impairment was in contact with the table for an average time of $4.2 \pm 3.1 \mathrm{~s}$, while for the unimpaired case the average time is $2.4 \pm 2.4 \mathrm{~s}$. Finally, the contact force was different for the experiments considered. In fact, mean value of norm of contact forces for the tactile impairment case is $23.2 \pm 8.6 \mathrm{~N}$, while is $12.3 \pm 5.7 \mathrm{~N}$ in the other case.

\section{CONCLUSIONS AND Future WORKS}

In this paper we have studied human behavior during ECE focusing on hand configurations and hand-environment exerted forces. We developed a multi modal experimental setup able to record both hand movements and exchanged forces between hand and a support table. We performed experiments with human healthy participants with and without tactile impairment asking randomly to grasp and move a common use object. We found that main hand configurations are pretty invariant while altering the tactile feedback. We believe that one relevant finding is that the cardinality of these configurations actually changes. In fact it seems that subjects with impaired conditions (similar to the case of soft robotic hands with rigid pads) prefer simpler planning approaches, i.e. sliding or power grasp, instead of precision grasp. This is further indication that tactile feedback increases the ability in precision grasps [22] and could suggest the introduction of sensorization strategies for robotic soft hands. Our claims are also supported by the reported force analysis. In fact, we noticed that subjects performing a task with impaired conditions exerted higher forces with the environment and need more time for its completion. Future works will focus on improving the clustering analysis to better classify human behavior and apply these results for robotic hand design and control.

\section{ACKNOWLEDGMENT}

This work is supported by the European Commission H2020 grants "WEARHAP" (no. 601165), "SOMA" (no. 645599) and "SOFTPRO" (no. 688857), and by the ERC Advanced Grant no. 291166 "SoftHands". 


\section{REFERENCES}

[1] M. Santello, M. Bianchi, M. Gabiccini, E. Ricciardi, G. Salvietti, D. Prattichizzo, M. Ernst, A. Moscatelli, H. Jörntell, A. M. Kappers et al., "Hand synergies: Integration of robotics and neuroscience for understanding the control of biological and artificial hands," Physics of life reviews, vol. 17, pp. 1-23, 2016.

[2] S. Casini, V. Tincani, G. Averta, M. Poggiani, C. D. Santina, E. Battaglia, M. G. Catalano, M. Bianchi, G. Grioli, and A. Bicchi, "Design of an under-actuated wrist based on adaptive synergies (accepted)," ICRA, 2017.

[3] S. Bruno and K. Oussama, Eds., Handbook of Robotics. Springer, 2016, no. 38, ch. Grasping.

[4] R. Deimel and O. Brock, "A novel type of compliant and underactuated robotic hand for dexterous grasping," The International Journal of Robotics Research, vol. 35, no. 1-3, pp. 161-185, 2016.

[5] M. G. Catalano, G. Grioli, E. Farnioli, A. Serio, C. Piazza, and A. Bicchi, "Adaptive synergies for the design and control of the pisa/iit softhand," The International Journal of Robotics Research, vol. 33, no. 5, pp. 768-782, 2014.

[6] C. Della Santina, M. Bianchi, G. Averta, S. Ciotti, V. Arapi, S. Fani, E. Battaglia, M. G. Catalano, M. Santello, and A. Bicchi, "Postural hand synergies during environmental constraint exploitation," Frontiers in Neurorobotics, vol. 11, p. 41, 2017.

[7] M. Bonilla, E. Farnioli, C. Piazza, M. Catalano, G. Grioli, M. Garabini, M. Gabiccini, and A. Bicchi, "Grasping with soft hands," in Humanoid Robots (Humanoids), 2014 14th IEEE-RAS International Conference on. IEEE, 2014, pp. 581-587.

[8] G. Averta, C. Della Santina, E. Battaglia, F. Felici, M. Bianchi, and A. Bicchi, "Unvealing the principal modes of human upper limb movements through functional analysis," Frontiers in Robotics and AI, vol. 4, p. 37, 2017

[9] E. Battaglia, M. Bianchi, A. Altobelli, G. Grioli, M. G. Catalano, A. Serio, M. Santello, and A. Bicchi, "Thimblesense: a fingertip-wearable tactile sensor for grasp analysis," IEEE transactions on haptics, vol. 9, no. 1, pp. 121-133, 2016.

[10] C. R. Mason, J. E. Gomez, and T. J. Ebner, "Hand synergies during reachto-grasp," Journal of Neurophysiology, vol. 86, no. 6, pp. 2896-2910, 2001.

[11] C. Eppner, R. Deimel, J. Álvarez-Ruiz, M. Maertens, and O. Brock, "Exploitation of environmental constraints in human and robotic grasping," The International Journal of Robotics Research, p. 0278364914559753 , 2015 .

[12] B. Schäling, The boost C++ libraries. Boris Schäling, 2011.

[13] A. Serio, M. Bianchi, M. Gabiccini, and A. Bicchi, "[d94] the tactile toolbox," in Haptics Symposium (HAPTICS), 2014 IEEE. IEEE, 2014, pp. 1-1.

[14] M. Santello, M. Flanders, and J. F. Soechting, "Postural hand synergies for tool use," The Journal of Neuroscience, vol. 18, no. 23, pp. 10105 $10115,1998$.

[15] M. Gabiccini, G. Stillfried, H. Marino, and M. Bianchi, "A data-driven kinematic model of the human hand with soft-tissue artifact compensation mechanism for grasp synergy analysis," in 2013 IEEE/RSJ International Conference on Intelligent Robots and Systems. IEEE, 2013, pp. 37383745 .

[16] A. Kapandji, "Clinical test of apposition and counter-apposition of the thumb," Annales de chirurgie de la main: organe officiel des societes de chirurgie de la main, vol. 5, no. 1, pp. 67-73, 1985.

[17] C. De Boor, C. De Boor, E.-U. Mathématicien, C. De Boor, and C. De Boor, A practical guide to splines. Springer-Verlag New York, 1978, vol. 27.

[18] V. Sircoulomb, G. Hoblos, H. Chafouk, and J. Ragot, "State estimation under nonlinear state inequality constraints. a tracking application," in Control and Automation, 2008 16th Mediterranean Conference on. IEEE, 2008, pp. 1669-1674.

[19] Q. Fu and M. Santello, "Tracking whole hand kinematics using extended kalman filter," in Engineering in Medicine and Biology Society (EMBC),
2010 Annual International Conference of the IEEE. IEEE, 2010, pp. 4606-4609.

[20] R. W. Schafer, "What is a savitzky-golay filter?[lecture notes]," IEEE Signal processing magazine, vol. 28, no. 4, pp. 111-117, 2011.

[21] A. Serio, M. Bianchi, M. Gabiccini, and A. Bicchi, "[d94] the tactile toolbox," in Haptics Symposium (HAPTICS), 2014 IEEE. IEEE, 2014, pp. $1-1$.

[22] R. Johansson and G. Westling, "Signals in tactile afferents from the fingers eliciting adaptive motor responses during precision grip," Experimental brain research, vol. 66, no. 1, pp. 141-154, 1987. 